\title{
Study on the Application of 3D Printing Technology on the Construction of Clothing Space Styling
}

\author{
Jia $\mathrm{Hu}^{1}$ \\ ${ }^{1}$ Jiangxi Institute of Fashion Technology, Nanchang, Jiangxi, 330201
}

\begin{abstract}
KEYWORDS: 3D Printing Technology; Clothing; Spatial Modeling; Construction;
\end{abstract} Recommendations

\begin{abstract}
Costume design needs to use a number of technical components and each have their own unique effect. Costume design is a market with a wide range of industries, our lives plays an important influence. Costume design is the soul of clothing, the clothing style in the use of technology to nature is also the largest and the most advanced. Especially in recent years, increasingly popular use of 3D printing technology to provide a more convenient costume patterns and more space for development. This article by the action of 3D technology in the clothing space modeling to analyze the impact generated by their clothing style, and for the future of 3D technology to provide some reference value in the use of the clothing industry.
\end{abstract}

\section{Introduction}

With the rapid economic development and the progress of science and technology, the development of the apparel industry is also showing a strong upward trend, especially the emergence of 3D printing technology and application, for the apparel industry innovation and progress offers more possibilities, their also a new era of a great performance apparel industry. The 3D printing technology in the clothing specific spatial modeling builds on the role of it? Then through a series of research and analysis in order to obtain, the next step is analyzed for various functions and features of 3D printing technology space modeling clothing and other factors.

\section{The Meaning of 3D Printing Technology}

The so-called 3D printing technology refers to the three-dimensional software to edit the image model made use of a particular print tool to be printed at different levels, eventually showing a physical model with 3D stereoscopic effect. The origin of this technology was first produced in 1983, it has a long and distinguished history. Information technology 3D printing process which produces a study and have purposes, mainly refers to the specific ways and means will have a three-dimensional visual effect of drawing a special kind of material formed to show up, which presented results is three-dimensional, is three-dimensional, virtual reality model by material and technology's demonstrated, this is the essence of 3D printing technology, which not only is a great invention, more of today's costume design provides a item important technical support. Clothing space Construction widely applied to the 3D printing technology, the garment industry is one of the important progress.

\section{The Construction Elements of Spatial Modeling Clothing}

Clothing style mainly refers to the use of designer clothing outside the body belongs to the three-dimensional space and the specific image with visual beauty and the role of special clothing 
apparel made by the use of composite material is formed, which is commonly known as the clothing styles. Clothing style elements involved in many of the most obvious of which is the structural design of space clothing. Clothing spatial modeling includes modeling of its internal space and external space modeling. Mainly refers to the internal space of the shape of the human body in close contact with that part of the shape of the space, its purpose and design of the main impact is into wearing comfort and practicality. In the space inside the shape, the comfort of designer clothing to the first, interior space design is mostly outside could not see, and more from the consumer personally try to feel it, from the point of view is more appropriate appropriateness of clothing; and build external space are much more aesthetic appearance construct. Costume designer in the external space design clothing, was considered more of a garment's overall image and its demonstrated aesthetic concepts and models. Most consumers in the purchase of clothing will first consider the shape of the outer space, to meet their aesthetic requirements. Fashion design major in the design of these two spaces on the start, the aesthetic function of clothing and apply function through the grasp of these two spaces completely unfolded.

\section{D Printing Technology is Recommended in Clothing Space Styling}

The shape of the internal space and external space modeling clothing to some extent, is a complementary relationship, study its specific circumstances, the internal space of the modeling is mainly to solve the inherent apparel available features, but the main problem is to solve the external space modeling clothing modeling beauty, these two points are clothing indispensable elements. To 3D printing technology will play a role in the clothing space modeling, it is necessary to these two aspects, the 3D printing technology printing technology flexibly applied to the inner space and outer space modeling clothing.

3D printing technology principle is to properly handle the coordination between the image and the spatial extent, through special techniques to shape and space combine to achieve a certain visual and apply effects. Clothing for the interior space of the composition, how it works and clothing to interior design 3D printing technology combined model design method of 3D printing technology can be used in clothing design into the complex process of fitting the model up, by model adjustments to achieve the internal space modeling clothing adjustments. This not only saves time spent in design, it is possible to improve the design accuracy. The 3D printing technology flexibly applied to the internal design of the clothing, the clothing provide technical guarantee for interior space design.

On the 3D printing technology used in clothing design exterior space modeling, it is also an important technology utilization. External space modeling clothing includes the relationship between its own and its exterior styling and the natural environment. The key lies in the shape of the external space to meet the aesthetic requirements of clothing, as well as different consumer groups with different clothing style requirements. Aesthetic design model 3D printing technology contained in the principles of architecture and space for the exterior design of clothing provides an effective reference. In addition to the basic costume design clothing outside help, 3D printing technology can also play a role in the clothing external decoration. 3D printing technology can use different models of clothing accessories reference is embedded, because the 3D model with realistic three-dimensional visual effects, so by seeing the different models of accessories in the clothing of the presentation of the different effects have larger reference, able to provide effective technical support for the external clothing accessories design, which enables the development of fashion design have a broader space for development, for the development of the apparel industry to provide more possibilities. 
Costume design and production included numerous technical components, and each component technology all have an irreplaceable role. Although 3D printing technology plays a huge role in fashion design, but it still can not replace other technical effect. Therefore, in order to better design better clothing, better integration of 3D printing technology used in clothing spatial modeling to build them, they must be combined with other technologies to form a complete system of technology used, and can not be neglected. Clothing apparel design space modeling to build the most important part, in order to effectively do this plate, then is sure to use a variety of techniques flexibly together, and make full use of their respective advantages, to provide technical support for the costume design. In addition, the garment manufacturing also need to have superb technology and good material to do support, with good technology does not mean that will be able to produce good clothing, good material is essential, and only in good material and delicate on the basis of technology, fashion design technology exists only basis.

\section{Conclusion}

3D printing technology plays a growing role and also provides some reference for the development of other sectors in space constructed clothing. 3D printing technology to be fully applied to the inner space and outer space design shape of the design of clothing, were comprehensively at all levels, not only at the technical level to provide support for costume design, practicality has also provided support for the costume design . Clothing modeling spatial structure is the most important issue to be solved in costume design, inputs technical content will also be up for the use of 3D printing technology, but also to devote more energy to carry out analysis and research will be actively 3D printing technology combined with other technologies, various technical features will make full use of them, to provide a more solid support for the design and manufacture of clothing. On this basis, but also have good material and sophisticated technology to pave the way, only with these conditions, a truly outstanding costume design. This 3D printing technology also has some reference in the future direction of development.

\section{Reference}

[1] Ju Lie. On "Spatial Modeling Clothing" Course Summary [J]. Education Article, 2011 (12).

[2] Yu Guanghua. Shape does not Know the Course, Students Touch - Space Books Incision Modeling Study [J]. Anhui Literature (Second Half), 2010 (05).

[3] Zhang Heng. 3D-Looksailorxt System [J]. On The Basis of Clothing Pattern Design. Changchun Institute of Technology (Natural Science). 2015 (03).

[4] Zhou Lihong. Single Collar Shape Parameters Affecting Analysis [J]. Zhejiang Textile \& Fashion College. 2015 (04).

[5] Tang Jiqun. Deng Meizhen. Ping Shrug Sleeves Fit Female Fashion Modeling Analysis and Applied Research to Explore. [J]. Zhejiang Textile \& Fashion College. 2016 (01).

[6] Wang Guohai. Zhejiang Textile Clothing School Students to "Gather The Number of" Interpretation of the Era of Big Data [J]. Textile and Apparel Weekly. 2015 (22). 